\title{
The VHP Model
}

\author{
Nancy L. Moureau
}

\begin{abstract}
More than $90 \%$ of patients admitted to acute care receive intravenous access for the delivery of treatment. The concepts of vessel preservation and risk reduction incorporate the topics that apply to all aspects of vascular access insertion and management for patient intravenous (IV) medical treatment. By following a specific clinical pathway of care that adheres to evidence-based practice, the outcomes are optimized, veins are preserved, and the treatment plan completed while minimizing delays and complications. VHP promotes patient-focused practices that reduce morbidity of untoward effects associated with intravenous devices.
\end{abstract}

\section{Keywords}

Vessel health - Vessel health and preservation Quadrants of care - Vascular access assessment and selection $\cdot$ Vein assessment Insertion · Care and management Vascular access evaluation

N. L. Moureau ( $\bowtie)$

PICC Excellence, Inc., Hartwell, GA, USA

Menzies Health Institute, Alliance for Vascular Access Teaching and Research (AVATAR) Group, Griffith University, Brisbane, QLD, Australia e-mail: nancy@piccexcellence.com

\subsection{Introduction to Vessel Health and Preservation}

Vessel health and preservation (VHP) is a model applied to vascular access and the administration of IV medications and treatment that structures evidence-based practices within four quadrants of medical care: assessment/selection, insertion, management, and evaluation of vascular access devices. The model incorporates evidence-based practices, guidelines, and recommendations from many countries to guide practice from patient admission through completion of treatment. Application of the VHP is designed to ensure a higher level of safety for the patient, reduce risk with device selection, limit negative consequences of insertion using specially trained clinicians, promote complication-free device longevity through proper care and maintenance practices, and complete the process by evaluating and removing devices as soon as treatment is completed. From the first quadrant to the last, evidence guides practice as implemented in education to clinicians providing care.

The aim of the VHP model and pathway is to improve quality of care, reduce risks associated with vascular access devices (VADs), and increase patient satisfaction and efficiency in the use of healthcare resources. The model is represented in a pathway within quadrants of care that follow the patient treatment process. A clinical pathway is a step-wise process for management 
of patient care that promotes efficiency within a defined group of patients, those requiring vascular access devices, during a defined period such as the treatment process (De Bleser et al. 2006; Hanchett and Poole 2001). The content of a clinical pathway is based on scientific evidence, research, recommendations, and professional consensus. Clinical pathways improve patient outcomes by reducing variability, adding standardization, and reducing medical errors (Panella et al. 2003).

Assessment is represented in the first quadrant of the pathway with proactive site selection as the first stage of the VHP model (Fig. 1.1). Ensuring that each device is clinically indicated and the lowest risk necessary for the treatment and duration intended is vital to the patient safety (Chopra et al. 2015). Following patient and vein assessment, stage two is intravenous device selection and insertion by a qualified clinician.

Quadrant two includes the insertion stage where the final selection of the type of device, number of lumens, insertion procedure, and most qualified inserter are selected. Study and evaluation of intravenous device size, length, type, complications, insertion procedures with outcomes, and patient risk factors are all vital to this stage. It is within insertion that visualization technologies are employed to promote the highest degree of success.

Establishing and maintaining a route of access to the bloodstream is essential for patients in acute care. Quadrant three encompasses maintenance of the device, which is the longest stage in the life of the catheter. The last stage of the VHP model involves discontinuation of the treatment and removal of the device, followed by evaluation of patient outcomes and clinician competency. Education for clinicians inserting and managing the devices is an overarching theme of the entire model and is incorporated into all four quadrants of care.

Each of these stages forms an intentional process to guide selection, insertion, management, and discontinuation of the vascular access device (VAD). Often medical facilities are driven by default actions, based on crisis management when a VAD fails, opting to quickly select and replace with another similar device rather than thinking through the patient-specific process of selection. The concepts of vessel preservation and risk reduction incorporate the problems and subjects that apply to all aspects of vascular access insertion and management for patient IV medical treatment. VHP establishes a framework or pathway that follows each step of the patient
Fig. 1.1 Vessel health and preservation: Four quadrants of care (used with permission

N. Moureau, PICC Excellence)

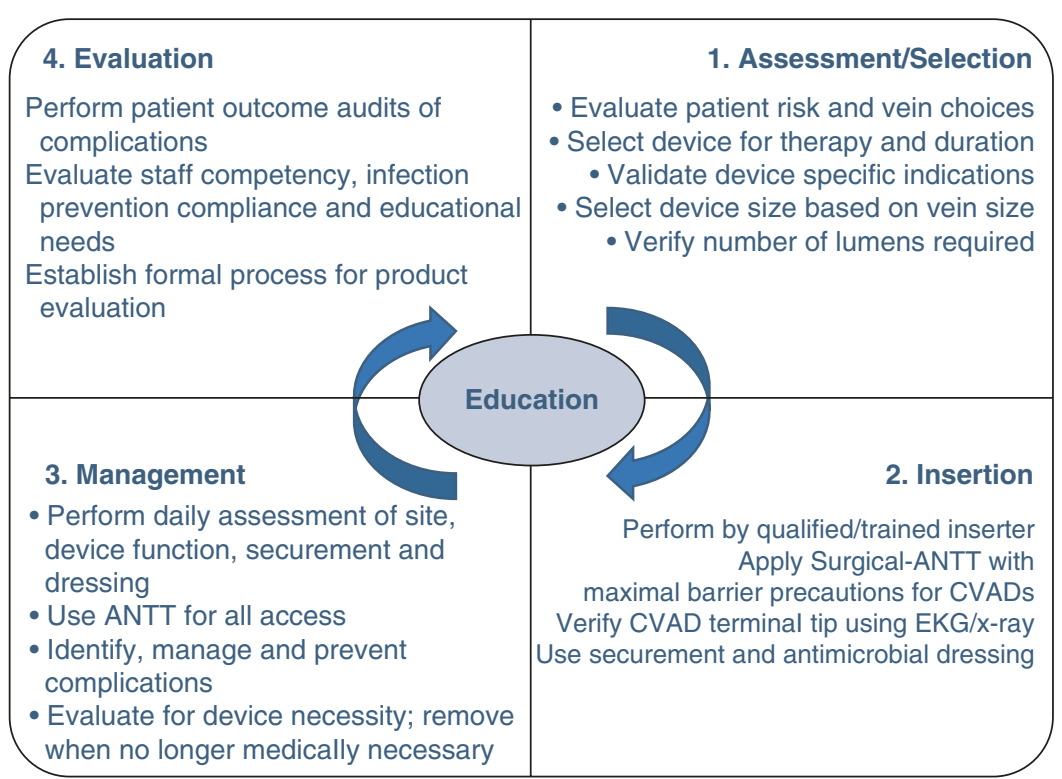


experience, intentionally guiding clinical care and improving and establishing a structure to reduce patient risk.

Comprehensive assessment and selection of the best vein and insertion location, performed by a highly skilled inserter using the most appropriate device, managed in a precise way, and removed at the right time is a process that requires commitment to education, policy development, and specialized clinicians. For facilities fortunate enough to have specialists or speciality teams performing insertion and assessment, the VHP process becomes intuitive. When bedside nurses, physicians, and others are responsible for single steps in the process, fragmentation results and the patient suffers (Castro-Sanchez et al. 2014; Moureau et al. 2012; Panella et al. 2003). The evidence suggests that reduction of fragmentation, by establishing a pathway and teaching a structured process to all stakeholders reduces complications with IV therapy, improves efficiency and diminishes cost (Gaddis et al. 2007; Gurzick and Kesten 2010; Hallam et al. 2016; Weston et al. 2017).

\subsection{Four Quadrants of Care}

\subsubsection{Quadrant 1: Right Assessment, Vein, and Device Selection}

Right assessment and selection of the best vein and location is the first stage of the vascular access cycle. This stage begins at the time of admission and continues as diagnosis is established. Most patients receive their first intravenous device hurriedly inserted in the emergency department; location and method of insertion are often not optimal. Once the patient has stabilized, consideration is given to the most appropriate vascular access device that will provide the administration of the prescribed therapy. Assessments of patient history, comorbidities, contraindications, available veins, diagnosis, and duration are factors that determine level of risk, the appropriate device, and most qualified inserter. Individuals with ultrasound training can apply these skills for assessment and selection of the right location and vein for device insertion.

\subsubsection{Quadrant 2: Right Insertion and Training}

Selection of the right device and the right inserter encompasses the second stage of the VHP process. Appropriate device selection and number of necessary lumens is a determination made according to lowest risk for patient insertion and potential for infection in conjunction with the needs of the therapy. Selection of the inserter and application of infection prevention principles are contributing factors for patient safety. Vascular access specialists and teams of specially trained clinicians function to aid in selection and insertion of the most appropriate device. Ensuring the insertion is performed by a trained and qualified clinician with ultrasound skills reduces insertion and post insertional complications.

\subsubsection{Quadrant 3: Right Management}

Management of vascular access devices represents the largest portion of time in the VHP cycle. Right management includes assessment of the insertion site, dressing, and device function prior to each infusion. Care and management using right infection prevention methods including Aseptic Non Touch Technique (ANTT) for device handling, disinfection of access site, pulsatile flushing the device before and after infusions, performing dressing changes consistent with policies, and evaluation of device necessity with prompt removal when the VAD is no longer needed are cornerstones to safe patient care. Incorporated into management are the right supplies and technology needed to ensure the right outcomes. Right management is a process that requires consistency established through commitment to education, policy development based on guidelines and research, and consistent evaluation of outcomes. 


\subsubsection{Quadrant 4: Right Evaluation}

Improvement of care is impossible without an established process of evaluation. Right evaluation for VHP program application includes outcome measurement of complications, observation of policy performance, and plan to provide education for staff departments and units with negative outcomes or practice deficiencies. A multimodal quality program applies guidelines and recommendations ensuring that practices are consistent and that staff are well informed through education and outcome reporting. Integrated with a VHP program is the evaluation of products, supplies, and technology, both existing and consideration for new product trial testing. Each product should undergo periodic assessment to determine performance according to the facility needs and expected application.

VHP quadrants all work together to deliver the highest quality of patient care through evidencebased practice. Patients receiving medical care should be able to trust for vascular access that:

1. The VAD selected has the lowest risk for insertion location, device size not to exceed $33 \%$ of vein diameter, length, and number of lumen, and is the most appropriate to deliver the treatment. The aim for the patient is greater comfort with reduction of the risk of complications.

2. Standard-ANTT or Surgical-ANTT is used for insertion, device management, dressing care, and medication administration with hub disinfection.

3. The VAD is individualized to patient-specific condition and medical history and placed in a suitable anatomical position to optimize dressing adherence and securement to minimize movement and reduce risk of premature failure.

4. The number of IV attempts is limited and will be performed by well-trained, qualified inserters supervised for competency.

5. The VAD is assessed for complications, dressing adherence, and flushed with normal saline to evaluate device function at least daily in acute care and removed when device is no longer needed, and treatment is complete.

6. The VAD is monitored and maintained by trained, competency-assessed clinical staff who receive consistent education on best practices for management of intravenous devices. Evaluation and education are provided on a timely and consistent basis to all clinical staff in connection with routine care and with any negative outcomes (assumes monitoring and reporting of all outcomes).

7. Concentrate medical product usage on those with scientifically studied and published evidence of positive patient outcomes.

Through the delivery of these seven trust points, patients maintain confidence in the healthcare system, avoid unnecessary costs and interventions, and achieve greater satisfaction with their healthcare centers all consistent with VHP precepts.

\section{Case Study}

A 250-bed acute care hospital identified quality gaps with negative outcomes associated with peripheral and central venous catheters. In an effort to reduce complications and improve patient outcomes, they committed to adopt the VHP model and apply it to all levels of care. The first step in the process was to provide training for patient assessment, followed by infection prevention education for all clinicians inserting or managing VADs. In addition, the education was continued to improve the competence of inserters to use ultrasound when needed, to implement better StandardANTT and Surgical-ANTT procedures, and to select the most appropriate device for the patient and therapy. Implementation of the VHP stages began in one targeted unit instituting initial assessment of every patient admitted to the area. Patient and clinician satisfaction feedback was collected and evaluated to identify improvement needs. Each patient received a detailed vascular access daily assessment that included evaluation of right device and right time if the device was still necessary, insertion site, dressing adherence, patient response, and 
catheter function. The final stage of the VHP process of implementation was evaluation of the outcomes and compliance with the process. While this hospital achieved a moderate $82 \%$ compliance with initial assessment, right inserter, daily assessments, and discontinuation of unnecessary VADs, their results had a huge impact on patient satisfaction and staff appreciation. Efficiency had improved with insertion changing from an average of 2.2 attempts with PIVCs to 1.4 per patient, supply usage was down 54\% with PIVCs changed only when clinically indicated, and even with the added staff time in VHP implementation, the savings continued to mount above $\$ 265,000$ estimated annual cost reduction.

\section{Summary of Key Points}

1. Vessel health and preservation (VHP) is a model applied to vascular access and the administration of IV medications and treatment that structures evidencebased practices within four quadrants of medical care: assessment/selection, insertion, management, and evaluation of vascular access devices.

2. Comprehensive assessment and selection of the best vein and insertion site location, performed by a highly skilled inserter using the most appropriate device, managed in a precise way, and removed at the right time is a process that requires commitment to education, policy development, and specialized clinicians.

3. Assessments of patient history, comorbidities, contraindications, available veins, diagnosis, and duration are factors that determine level of risk, the appropriate device, and most qualified inserter.

4. Ensuring the insertion is performed by a trained and qualified clinician with ultrasound skills reduces insertion and post insertional complications.

5. Device management includes assessment of the insertion site, dressing, and device function prior to each infusion.

6. Improvement of care is impossible without an established process of evaluation.

\section{References}

Castro-Sanchez E, Charani E, Drumright LN, Sevdalis N, Shah N, Holmes AH. Fragmentation of care threatens patient safety in peripheral vascular catheter management in acute care-a qualitative study. PLoS One. 2014;9:e86167.

Chopra V, Flanders SA, Saint S, Woller SC, O'Grady NP, Safdar N, Trerotola SO, Saran R, Moureau N, Wiseman S, Pittiruti M. The Michigan Appropriateness Guide for Intravenous Catheters (MAGIC): results from a multispecialty panel using the RAND/UCLA appropriateness method. Ann Intern Med. 2015;163(6 Suppl):S1-40.

De Bleser L, Depreitere R, Waele KD, Vanhaecht K, Vlayen J, Sermeus W. Defining pathways. J Nurs Manag. 2006;14(7):553-63.

Gaddis GM, Greenwald P, Huckson S. Toward improved implementation of evidence-based clinical algorithms: clinical practice guidelines, clinical decision rules, and clinical pathways. Acad Emerg Med. 2007;14:1015-22.

Gurzick M, Kesten KS. The impact of clinical nurse specialists on clinical pathways in the application of evidence-based practice. J Prof Nurs. 2010;26:42-8.

Hallam C, Weston V, Denton A, Hill S, Bodenham A, Dunn H, Jackson T. Development of the UK Vessel Health and Preservation (VHP) framework: a multi-organisational collaborative. J Infect Prev. 2016;17:65-72.

Hanchett M, Poole SM. Infusion pathways: planning for success. J Vasc Access Devices. 2001;6(3):29-37.

Moureau N, Trick N, Nifong T, Perry C, Kelley C, Carrico R, Leavitt M, Gordon S, Wallace J, Harvill M, Biggar C, Doll M, Papke L, Benton L, Phelan D. Vessel health and preservation (Part 1): a new evidence-based approach to vascular access selection and management. J Vasc Access. 2012;13:351-6.

Panella M, Marchisio S, Di Stanislao F. Reducing clinical variations with clinical pathways: do pathways work? Int J Qual Health Care. 2003;15:509-21.

Weston V, Nightingale A, O'loughlin C, Ventura R. The implementation of the Vessel Health and Preservation framework. Br J Nurs. 2017;26:S18-22. 
Open Access This chapter is licensed under the terms of the Creative Commons Attribution 4.0 International License (http://creativecommons.org/licenses/by/4.0/), which permits use, sharing, adaptation, distribution and reproduction in any medium or format, as long as you give appropriate credit to the original author(s) and the source, provide a link to the Creative Commons license and indicate if changes were made.

The images or other third party material in this chapter are included in the chapter's Creative Commons license, unless indicated otherwise in a credit line to the material. If material is not included in the chapter's Creative Commons license and your intended use is not permitted by statutory regulation or exceeds the permitted use, you will need to obtain permission directly from the copyright holder. 